\title{
Clinical Stem Cell Imaging and In vivo Tracking
}

\author{
Sahar Mirpour and Ali Gholamrezanezhad \\ The Russell H. Morgan Department of Radiology and Radiological Science, \\ Johns Hopkins Medical Institutions \\ USA
}

\section{Introduction}

The field of stem cell biology and regenerative medicine is rapidly moving toward translation to clinical application (Gholamrezanezhad, Bagheri et al. 2007; Gholamrezanezhad, Mirpour et al. 2011). Stem cells are able to perform restorative functions in patients with different disorders and hence stem cell therapy may be a potential alternative to some of the current modalities of treatment, such as organ transplantation. In fact, Cell transplantation therapy offers a means to stimulate tissue repair either by direct (graft-induced) or indirect (host-induced) tissue regeneration or angiogenesis (Kraitchman and Bulte 2009). During the past years, there has been enormous progress in understanding multipotent and pluripotent stem cells and their possible applications in clinical settings (Jang, Ye et al. 2011). After preliminary observations of the benefits of these cells in preclinical studies of different human disorders, the first clinical studies using bonemarrow-derived stem cells were initiated (Kraitchman and Bulte 2008). At this instant, stem cell-based therapies have been explored to potentially regenerate healthy tissues and it is clear that they can be the source of regenerate cells for different disorders (Jang, Ye et al. 2011). Although to-date numerous trials have been reported, yet there are remarkable debates on the efficacy of stem cell and the optimal treatment regime.

Many aspects of stem cell biology and their behavior inside of the human body are still beyond our understanding. One of the main obstacles and barriers in front of clear perception of the fate of stem cells in human body is lack of a perfect and ideal tool of cell tracking in patients receiving stem cells of different origin. In fact, it has been challenging to study developmental potentials of stem cells because they reside in complex cellular environments and aspects of their distribution, migration, engraftment, survival, proliferation, and differentiation often could not be sufficiently elucidated based on limited snapshot images of location or environment or molecular markers. Therefore, reliable imaging methods to follow or track the outcome of stem cells are highly required (Kraitchman and Bulte 2008; Jang, Ye et al. 2011; McColgan, Sharma et al. 2011), in order to confirm adequate homing of the injected cells to the target organ. The cell fate can be verified in animal models using histopathologic examination of tissue. However, in clinical settings and in human studies, noninvasive methods for assessment of cell survival and engraftment will be needed to assess therapeutic efficacy. Like detecting cells microscopically, cell labeling for noninvasive in vivo imaging relies on targeting contrast agents to stem cells to enhance their conspicuity relative to local tissue (Kraitchman and 
Bulte 2009). Both short- and long-term monitoring of stem cells in cultures and in living organisms have benefited from recently developed imaging techniques which are applied to assess cell behavior and function. Confocal and multiphoton microscopy, time-lapse imaging technology, transfection with reporter genes and also noninvasive clinical imaging modalities have provided the opportunity to monitor cell behavior in the context of a living organism. In turn, the knowledge gained through these data has brought the understanding of stem cell behavior to a new stage (Jang, Ye et al. 2011).

"Successful in vivo imaging requires that a contrast agent associated with a stem cell exert an "effect size" sufficient for detection by imaging hardware. Although the most attractive contrast agents for tracking are endogenous ones (ie, normal components of the stem cell), their effect size is extremely small" (Frangioni and Hajjar 2004). Frangioni et al described the 8 characteristics of an ideal imaging technology for stem cell tracking, among them biocompatibility and safety of the exogenous contrast agent was the first, and foremost factor. No need for genetic modification or perturbation to the stem cells, single-cell detection at any anatomic location, quantification of cell number, minimal or no dilution with cell division, minimal or no transfer of contrast agent to nonstem cells, noninvasive imaging in the living subject over months to years, and no requirement for injectable contrast agent were the other characteristics (Frangioni and Hajjar 2004). Unfortunately most of these features are not fulfilled by the currently applied methods of clinical in vivo stem cell tracking for human trials or daily clinical practice.

\section{A. Techniques of Stem Cell Imaging}

Among the different methods examined in clinical settings, radioactive labeling with radiopharmaceuticals and magnetic resonance imaging (MRI) are the most widely used methods for tracking infused stem cells, and therefore will be described in more details. However, the current noninvasive imaging approaches for tracking stem cells in vivo include also imaging with quantum dots (QDs), and reporter genes. We will review all the current imaging modalities and evaluate their relative advantages versus disadvantages. Because no single contrast agent/detector pair will satisfy all essential requirements of stem cell tracking in clinical settings, dual- and multimodality techniques, which combine the best features of each technology, have been recommended (Josephson, Kircher et al. 2002; Hill, Dick et al. 2003; Wickline and Lanza 2003; Doubrovin, Serganova et al. 2004; Frangioni and Hajjar 2004; Wu 2007; Zhang and Wu 2007; Higuchi, Anton et al. 2009; Gera, Steinberg et al. 2010).

\section{Plain radiography and computed tomography}

Plain $\mathrm{X}$ ray radiography and computed tomography $(\mathrm{CT})$ are the most readily available clinical imaging modalities with low cost. In order to obtain images with optimum quality on plain radiographs or $\mathrm{CT}$ and to render stem cells visible, contrast generation requires extremely high concentrations of high-density/high-atomic number materials (e.g. iodine, gadolinium, or metals). Such contrast is difficult to achieve in clinical settings, rendering these methods unlikely to play a direct role for stem cell tracking purposes (Frangioni and Hajjar 2004).

\section{Nanoparticle labeling}

Semiconductor quantum dots (QDs), as new class of fluorescent probes, has been applied in noninvasive imaging, using fluorescent semiconductor nanocrystals to detect membrane 
molecules of interest (Jaiswal, Mattoussi et al. 2003; Lin, Xie et al. 2007; Zhang and Wu 2007; Lu, Xu et al. 2010; Pi, Zhang et al. 2010; Ramot, Steiner et al. 2010). Depending on the size and composition of these probes, they can be designed to emit different wavelengths of light, ranging from ultraviolet to nearly infrared. Although there are minimal experiences and published reports with their application for the purpose of in vivo stem cell tracking (Lu, Xu et al. 2010; Yukawa, Kagami et al. 2010), the photostability of QDs, as seen in their resistance to photobleaching and long-lasting fluorescence, suggest them as attractive tools for tracking stem cells in vivo (Lin, Xie et al. 2007; Zhang and $\mathrm{Wu}$ 2007). However, some concerns about the effects of QDs on stem cell proliferation and differentiation (Hsieh, Wang et al. 2006) and safety of this approach have been raised (Ramot, Steiner et al. 2010). Fortunately, recent evidences are negative regarding any adverse effect of QDs on viability, proliferation, and pluripotency of stem cells as compared with non-labeled control cells (Ruan, Shen et al. 2010; Rutten, Janes et al. 2010). Also some other obstacles, including the tendency for aggregation of QDs in the cytosol, impenetrability of QDs into cells, nonspecific binding to multiple molecules, and degradation or excretion of QDs must be overcome before QDs can realize their full potential in bioimaging and clinical settings (Zhang and Wu 2007; Pi, Zhang et al. 2010). Among different QDs suggested for the purpose of stem cell tracking, MNPs@SiO2(RITC) seems to be promising, as they offer advantages of photostability against ultraviolet light exposure, nontoxicity to human stem cells, do not affect the surface phenotype or morphology of human stem cells, and also have stable retention properties in stem cells in vitro. These results demonstrate that MNPs@SiO2(RITC) are biocompatible and useful tools for human MSC labeling and bioimaging. Moreover, MNPs@SiO2(RITC) have multimodal fluorescent and magnetic characteristics. Furthermore, Park et al using optical and magnetic resonance imaging, successfully detected a visible signal from labeled stem cells that were transplanted into mice (Park, Tae et al. 2010).

\section{Reporter gene labeling}

Reporter gene imaging has introduced as an exceptional tool to localize specific molecules of interest within living subjects (Higuchi, Anton et al. 2009; Kraitchman and Bulte 2009). The reporter gene of interest usually encodes a specific protein, which encompass the potential to interact with an imaging probe and generating signals captured and quantified by conventional imaging modalities, such as SPECT, PET, MRI, or an optical charge-coupled device (Zhang and $\mathrm{Wu}$ 2007). Although it has been noted that "these imaging techniques can be used to assess cell trafficking with methods that are easily translatable to humans" (Acton and Zhou 2005), several shortcoming (which are discussed later) should be overcome before reaching to clinical applications.

\section{Optical imaging}

Bioluminescence and fluorescence are two complementary optical imaging techniques which are used for stem cell tracking. Bioluminescence utilizes light generated by the enzyme luciferase to detect cells in vivo, the effectiveness of which has been confirmed in small animal models (Tang, Shah et al. 2003; Wang, Rosol et al. 2003; Wu, Chen et al. 2003; Cao, Wagers et al. 2004; Frangioni and Hajjar 2004; Min, Ahn et al. 2006; Bradbury, Panagiotakos et al. 2007; Min, Le et al. 2008; Reumers, Deroose et al. 2008; Zhang, Qiao et al. 2011). Unfortunately, luciferase genes and other candidate substrates described to date 
generate only visible light (spectrum: 400 to $700 \mathrm{~nm}$ ), which has very high absorption and scatter in living tissue, precluding its application in animals larger than rats [6]. The need to stable expression of nonhuman genes and the injection of high concentrations of potentially immunogenic, nonhuman substrates, (e.g. luciferin and coelenterazine) are the other limitations of the technique. It is therefore unlikely that bioluminescence reaches clinical application [6].

Fluorescence imaging, another widely used technique of optical imaging, uses a fluorescent protein for in vivo imaging (eg, green fluorescent protein, small-molecule polymethines) or organic/inorganic hybrids (eg, quantum dots) (Frangioni and Hajjar 2004; Zhang and Wu 2007). Near-infrared fluorescence imaging has decreased absorption and scatter of photons at 700 to $1000 \mathrm{~nm}$, which is advantage of the technique over bioluminescence. However, similar to bioluminescence, near-infrared fluorescence imaging is limited to a shallow tissue depth $(4-10 \mathrm{~cm})$, even with tomographic imaging methods. Hence, clinical application of fluorescence imaging likely will be restricted to near-surface imaging, such as intraoperative procedures (Reynolds, Troy et al. 1999; Tepper-Wessels, Loeschinger et al. 2001; Nakayama, del Monte et al. 2002; Bonde, Maxwell et al. 2005; Sung, Hong et al. 2009; Polzer, Haasters et al. 2010). Similar to scintigraphic and MRI techniques of stem cell tracking, major disadvantages of fluorescence imaging are the dilution of the agent with each cell division and the possibility of uptake by non-stem cells following stem cell death (Frangioni and Hajjar 2004; Zhang and Wu 2007).

Several studies have confirmed the effectiveness and practicability of reporter gene labeling with bioluminescence and fluorescence markers to track the distribution and engraftment of stem cells in animal experiments using optical imaging (Cao, Lin et al. 2006; Sheikh, Lin et al. 2007; Zhang and Wu 2007; Li, Suzuki et al. 2008). Although the signals generated by these techniques are more sensitive than those of other imaging modalities in small animals, their emissions can easily be scattered within deep tissues. Hence, this current inability to accurately determine cell depth is a major limitation (Zhang and $\mathrm{Wu} 2007$ ).

\section{Ultrasound}

Regarding the wide availability and easy applicability of ultrasound and echocardiography, tracking by these techniques would be extremely convenient. In these techniques, contrast for echocardiography is generated by acoustic interfaces such as water/gas (eg, microbubbles, perfluorocarbons) (Frangioni and Hajjar 2004; RodriguezPorcel, Gheysens et al. 2005). "Although a single unit of contrast is on the order of 0.25 to $1 \mu \mathrm{m}$ in diameter, the generated acoustic perturbation appears much larger" (Frangioni and Hajjar 2004). Therefore, theoretically ultrasound-based techniques have the potential to detect a single cell loaded with a single unit of contrast (Frangioni and Hajjar 2004; Klibanov, Rasche et al. 2004). However, techniques to intracellular ultrasound contrast labeling are not yet robust, and more importantly, their possible effects on cell function are not yet identified. Exactly similar to MRI and scintigraphic techniques of stem cell tracking, contrast agents are subject to dilution during cell division and transfer to nonstem cells following leakage out of the cells or cell death. Another shortcoming of echogenic contrasts is the possibility of casting acoustic "shadow" below the first unit of contrast detected, which in conjunction with low spatial resolution of ultrasound and its inaccessibility in many anatomic sites, theoretically precludes precise quantification of cell number (Frangioni and Hajjar 2004). 


\section{Scintigraphic tracking}

Scintigraphic techniques represents the commonest method of human therapeutic cell tracking and are better suited for whole-body biodistribution studies, as compared with iron oxide labelling for MRI (Detante, Moisan et al. 2009; McColgan, Sharma et al. 2011).

\section{- SPECT (Single Photon Emission Computed Tomography)}

Two approaches for in vivo stem cell tracking by nuclear medicine technology (SPECT or PET) have been described: 1) direct loading with a radiotracer, and 2) enzymatic conversion and retention of a radioactive substrate or receptor-mediated binding (Frangioni and Hajjar 2004).

a. Direct loading: For direct loading, three well-established cell labeling radiopharmaceuticals are ${ }^{18} \mathrm{~F}-\mathrm{FDG}$ (discussed in the section of PET), $99 \mathrm{mTc}-\mathrm{HMPAO}$ and 111In-Oxine, with short, intermediate and long half-lives, respectively. The time period during which radiolabeled cell distribution can be tracked scitigraphically is limited by the half life and decay of their label (Steindler 2007).

i. 99mTc-HMPAO: Technetium $(99 \mathrm{mTc})$ exametazime is a radiopharmaceutical routinely used for the labeling of leucocytes to localize infections and inflammatory bowel diseases (Weldon, Joseph et al. 1995). Because of its short half life (almost 6 hours) and its gamma ray energy well adapted to conventional gamma-cameras (thus ensuring high image quality), $99 \mathrm{mTc}-\mathrm{HMPAO}$ is well suited for short tracking of stem cells in vivo (even better than ${ }^{111} \mathrm{In}$ ) (Meyerwittkopf, Bockisch et al. 1993; Detante, Moisan et al. 2009). As a lipophilic complex that is reduced into a hydrophilic complex by a glutathione-dependent mechanism following entrapment in cells, $99 \mathrm{mTc}-\mathrm{HMPAO}$ provides a stable labeling marker for in vivo cell tracking (Neirinckx, Burke et al. 1988), and therefore it has been already used on different cell types to study cell biodistribution over periods of up to 24 hours (Detante, Moisan et al. 2009).

ii. 111In-oxine: Indium-111 is an Auger electron emitter, which internalizes nonspecifically into both normal and malignant cells (Bindslev, Haack-Sorensen et al. 2006; Gholamrezanezhad, Mirpour et al. 2009). Thanks to the long half life of Indium-111, serial tracking of cells over 10 days using single photo electron computed tomography (SPECT) imaging is possible (Gholamrezanezhad, Mirpour et al. 2011). Given that Indium-111 oxine was approved for clinical application for labeling white blood cells to track sites of inflammation since more than 20 years ago (Lavender, Goldman et al. 1977; Kraitchman and Bulte 2009), it was an acceptable extension to label stem cells for noninvasive tracking of cells in human biodistribution studies (Lavender, Goldman et al. 1977; Gao, Dennis et al. 2001; Aicher, Brenner et al. 2003; Barkholt, Danielsson et al. 2003; Chin, Nakamoto et al. 2003; Barkholt, Danielsson et al. 2004; Brenner, Aicher et al. 2004; Maskali, Tran et al. 2004; Maskali, Tran et al. 2005; Bindslev, Haack-Sorensen et al. 2006; Legrand, Cougnenc et al. 2007). Based on the Andersson et al. study, the decline of intracellular ${ }^{111}$ In concentration is most prominent during the first 6 hours postlabeling and appears to remain stable thereafter (Andersson, ForssellAronsson et al. 1996). Hence, the radiotracer seems to be suitable for clinical imaging, regarding its in vivo stability. In our experience in patients with liver cirrhosis, we found no unexpected adverse events or complications during labeled stem cell infusion and within one month follow up period (Gholamrezanezhad, Mirpour et al. 2011). The 
cells can be visualized in vivo up to at least 10 days after initial infusion and it is possible to quantify and follow them using radio-labeling with 111Indium derivatives (Gholamrezanezhad, Mirpour et al. 2011).

The main problem with application of 111 Indium-labed chemicals for stem cell tracking is the concern about its cytotoxic effects on stem cells which will adversely affect their possible therapeutic potential for patients. As the first experiment on human stem cells, we designed a study to continuously pursue the effect of Indium-111 labeling on stem cell viability during the 2-week period of post-labeling. After culturing mesenchymal stem cells (MSCs), we divided the cells into six samples, each of which contained $1 \times 10^{6}$ MSCs. The first sample was considered as the control. The remaining five samples (samples 2-6) were labeled with the following doses of ${ }^{111} \mathrm{In}$-oxine, respectively: 0.76, 1.64, 3.48, 5.33, and 7.16 MBq/106 MSCs. To evaluate the effects of ${ }^{111}$ In-oxine labeling on cellular viability and count, all samples were examined immediately after labeling ( $2 \mathrm{~h}$ ) as well as $24,48 \mathrm{~h}$, and 5, 7, and 14 days post-labeling (Gholamrezanezhad, Mirpour et al. 2009). Fortunately, no statistically significant relationship was found between labeling efficiency and administered dosage of radiopharmaceutical. In fact, the labeling efficiency showed no reduction with lower doses of the radiotracer applied to the incubation media. However, a significant inverse relationship was noted between radiotracer dose and viability of stem cells during the 2week period of follow-up. For example, with the specific activity of $4.98 \mathrm{MBq} / 10^{6} \mathrm{MSCs}$, more than $80 \%$ of the cells lost their viability within 2 weeks of follow-up. The cytotoxicity feature with the lower doses was much less (between $20 \%-60 \%$ ), but never was equal to zero. Hence, in our opinion, one of the main disadvantages inherent to ${ }^{111} \mathrm{In}$-oxine labeling of stem cells for the purpose of in vivo tracking is its time and dose dependent side effects. Although it has been shown that radio-labeling is one of the most sensitive methods for stem cell tracking, we recommended that the application of this tracking technique should be treated with great caution, and if necessary, as little of 111In-oxine as possible should be added to the cells (or only a limited fraction of the cells should be labeled) to minimize cell loss. Such a conclusion is also confirmed by other similar reported experiments (Correa, Mesquita et al. 2007). Hence, in our second experience to track stem cells after intravenous infusion to patients with liver cirrhosis [3], we just labeled $50 \%$ of the stem cells. Labeled cells were infused from one arm of patients and the non-labeled cells were infused from the opposite arm to reduce any possible adverse effects. Also the labeling dose was kept to the lowest possible, providing good-quality images [Fig. 1]. Such a concern also persists when using technetium derivatives, although with much less severity. In Detante et al experiment, 99mTc labeling (even at low doses of $5 \mathrm{~Bq} /$ cell) induced a loss of stem cells' ability to form colonies. However, they found no deleterious effect on cell proliferation with HMPAO alone and therefore they concluded that loss of stem cells' proliferation seems to be due to the 99mTechnetium. Although their results of flow cytometry with 7-AAD demonstrated a good cell viability over the 2 days of experiment, the authors emphasized that the alteration of the ability of radiolabeled stem cells to proliferate must be carefully considered, as it may alter their therapeutic benefit (Detante, Moisan et al. 2009).

It also should be stated that although nuclear medicine techniques to track stem cells following injection or infusion to patients are highly helpful and lack some of the remarkable disadvantages inherent to other modalities (such as low sensitivity of MRI), they suffer from technique-specific shortcomings. These shortcomings seem to be more prominent when the technique is applied in clinical and in vivo settings. It is possible that stem cells labeled with radiotracers are phagocytosed by the reticuloendothelial cells. We 


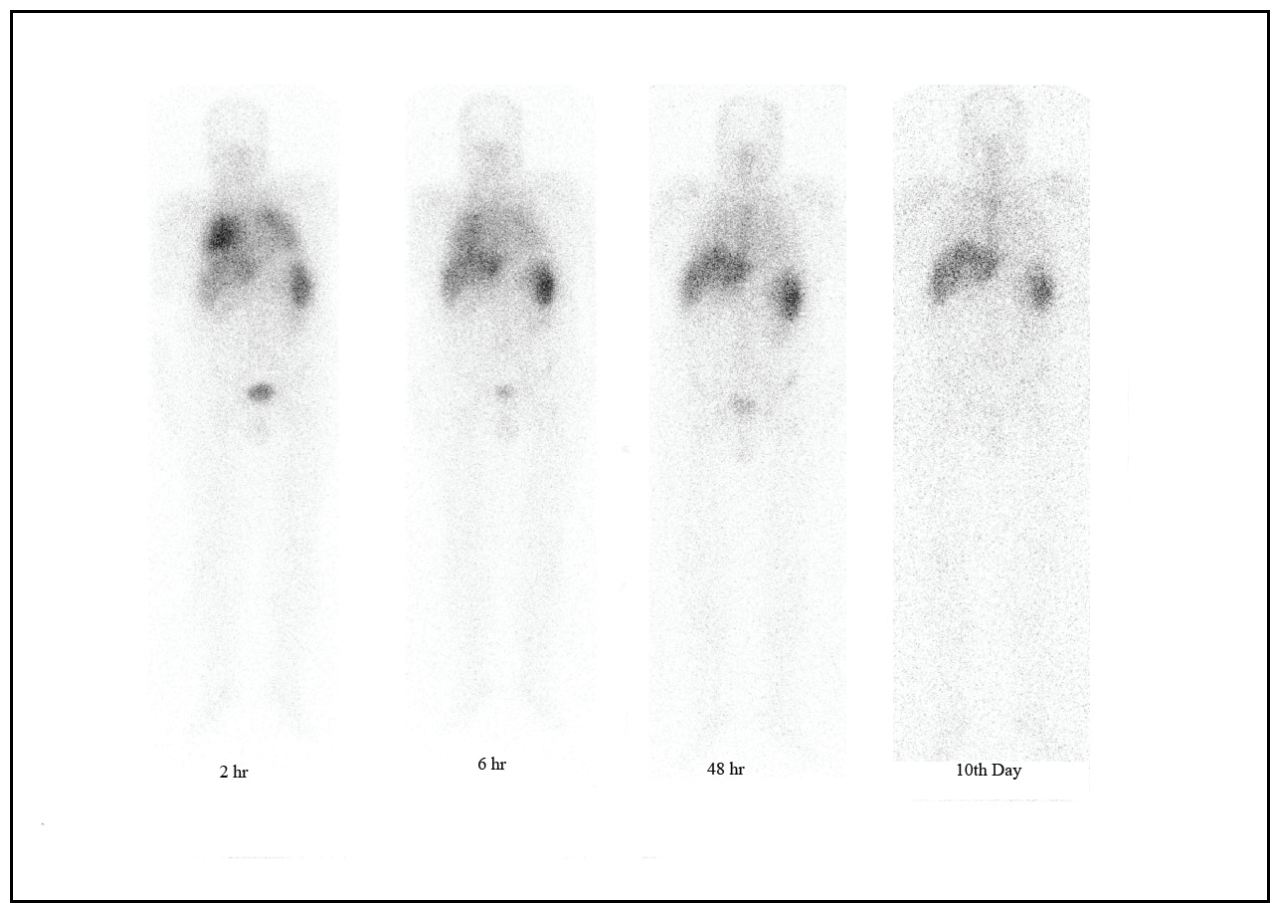

Fig 1. After I.V. infusion, the radioactivity was first observed to accumulate in the lungs, and gradually shifted to the liver and spleen. In this patient the initial hepatic accumulation of radioactivity was visualized at the second-hour images. Also, the early images ( $2 \mathrm{~h})$ demonstrated faint 111In activity in the spleen. During the following hours to days, the radioactivity became more prominent in the liver and spleen.

cannot exclude the possibility that radionuclide signals are detected from the reticuloendothelial cells, which theoretically are able to phagocytize nonviable stem cells (for example, due to time-dependent cytotoxic effect of the 111In-oxine, after infusion, a limited portion of stem cells will lose viability (Gholamrezanezhad, Mirpour et al. 2009). In fact, no one can be sure that the observed radioactivity in vivo reveals the presence of viable stem cells in the organs having radioactivity. However, it should be emphasized that this limitation is not limited to scintigraphic tracking methods: MR tracking of stem or other cells has a critical flaw that the ferromagnetic particles do not represent viable cells in vivo. We simply do not know whether MR signals mean magnetic particles in the "live" cells or "already dead" cells or even particles in the engulfed/digested cells. While label detachment from the cell is a primary concern for direct labeling techniques, similar issues exist with reporter gene imaging, where the reporter gene product may still be present after the cell has died (Gilad, Winnard et al. 2007). In other words, direct loading is problematic given the tradeoff between half-life and long-term exposure to inherently cytotoxic ionizing radiation and given the possibility of transfer of the radiolabel from stem cells to nonstem cells (Frangioni and Hajjar 2004).

Technical consideration: Region of Interest (ROI) analysis is a simple tool to measure the percentage of cells homing to the target organ. Decay and background corrections and 
geometric mean calculation are techniques to improve the reliability and accuracy of the data (Gholamrezanezhad, Mirpour et al. 2011). In animal studies, biodistribution analysis is possible, in which, organ, blood, and urine activities of labeled stem cells are expressed as count per minute (cpm), and then decay-correction to the time of injection, and normalization to the tissue weight (gram) and to the injected dose is done. Simply, these results can be expressed as percent of the injected dose per gram of sample weight (\%ID/g) (Detante, Moisan et al. 2009).

b. Enzymatic conversion/retention and Receptor-mediated targeting methods:

As fully explained by Zhang et al, these methods have been applied with both scintigraphic [SPECT and PET studies] and MRI substrates (Zhang and Wu 2007). Hence, the following features can be applied to both MRI and scintigraphic techniques: Receptor-mediated targeting needs stable expression of a receptor not found elsewhere throughout the body and intravenous injection of a labeling agent (Acton and Zhou 2005; Serganova and Blasberg 2005; Shah 2005; Belmar, So et al. 2007; Zhang and Wu 2007; Roelants, Labar et al. 2008; Higuchi, Anton et al. 2009; Kraitchman and Bulte 2009; Nyolczas, Charwat et al. 2009; Pomper, Hammond et al. 2009). For example, inducible promoters can be employed to modulate the level of reporter and therapeutic gene expression. Sodium iodide symporter gene for reporter gene imaging by I-124-PET has been also suggested (Higuchi, Anton et al. 2009). Tissue-specific promoters may also be employed as sensors of stem cell differentiation (Klug, Soonpaa et al. 1996; Zhang and Wu 2007). The noteworthy advantages of this technique are the ability to follow stem cells indefinitely after stable integration of the transgene, no marker dilution by cell division, and the ability to destroy stem cells by administration of a suicide drug specific for the enzyme. The disadvantages are the necessity to genetically manipulate stem cells ex vivo and intravenous administration of another dosage of labeling agent for each imaging session (Zhang and Wu 2007). Before reporter gene imaging can fully and safely be applied clinically, several problems must be solved, the main example of which are transfection stability, minimizing immunologic response and minimizing the potential interference of stem cell function and differentiation by vector transfection or transduction (Chung, Andersson et al. 2002; Li, Fehse et al. 2002; Jakobsson, Rosenqvist et al. 2004; Zhang and Wu 2007; Olmer, Haase et al. 2009; Tran, Lair et al. 2010).

Receptor-mediated targeting, which is briefly described previously, requires stable expression of a receptor not found elsewhere throughout the body and intravenous injection of a labeling ligand of the receptor.

\section{- Positron Emission Tomography (PET)}

Due to its high spatial resolution and high sensitivity, PET can be considered a high-quality imaging tool for stem cell tracking (Kang, Kang et al. 2006). ${ }^{18 F-F D G ~ h a s ~ b e e n ~ c o n s i d e r e d ~ a ~}$ candidate radiotracer for labeling stem cells. As glucose uptake is a physiologic process, uptake by stem cells does not need any cellular modification and occurs in vitro in the same manner as in vivo without specific manipulation (Tamura, Unno et al. 2004). Few studies have indicated that stem cells labeled with $18 \mathrm{~F}-\mathrm{FDG}$ could be used to monitor cell homing into the target tissues and to track the biodistribution of the injected cells (Hertenstein, Wollert et al. 2004; Tamura, Unno et al. 2004; Wollert, Hofmann et al. 2004; Hofmann, Wollert et al. 2005).

18F-FDG [2-deoxy-2-(18F)fluoro-D-glucose], a glucose analog with the positron-emitting radioactive isotope fluorine- 18 substituted for the normal hydroxyl group at the 2 ' position 
in the glucose molecule, is the most widely used label for the purpose of stem cell tracking. The radioactivity elimination half-life of 110 minutes.

Overall, PET has the advantage of the higher sensitivity than SPECT, which allows more accurate quantification of cell number (Frangioni and Hajjar 2004). Although both strategies mentioned above for SPECT stem cell tracking can be used for stem cell tracking with PET, the most advanced by far has been stated to be the stable integration of a mutant herpes simplex type 1 thymidine kinase (TK) into stem cells and periodic intravenous injection of the TK substrate 18FHBG (Wu, Chen et al. 2003; Frangioni and Hajjar 2004). The main advantage of the technique is its ability to track and quantify stem cells over the course of months; however disadvantages such as necessity of genetic manipulation of stem cells, an infrastructure for $18 \mathrm{~F}$ chemistry, a PET scanner, and concerns about toxic effects of radiation exposure to the stem cells and subject (albeit it intermittent) should be kept in mind (Frangioni and Hajjar 2004).

Also other shortcomings inherent to both SPECT and PET-based tracking of stem cells, which are comprehensively discussed by Frangioni and Hajjar, are: nonspecific uptake of the radiotracer by normal tissue, relatively low efficiency of collimated SPECT cameras, and photon attenuation by tissue (Frangioni and Hajjar 2004). Although tissue photon attenuation correction is currently employed very easily and brings the advantage of coregistering anatomic (CT) and physiologic (SPECT or PET) data, it can theoretically reduce sensitivity, and prevents accurate quantification of stem cell number (Chin, Nakamoto et al. 2003; Frangioni and Hajjar 2004) and also increases the radiation burden to the subject and also the cost.

\section{MRI}

MRI and magnetic resonance spectroscopy are common imaging modalities for in vivo stem cell tracking in both preclinical and clinical settings (Hoehn, Kustermann et al. 2002; Modo, Cash et al. 2002; van den Bos, Wagner et al. 2003; Kustermann, Roell et al. 2005; Crowder, Brant et al. 2006; Jansen, Shamblott et al. 2006; Au, Lam et al. 2007; Hsiao, Tai et al. 2007; Magnitsky, Walton et al. 2007; Partlow, Chen et al. 2007; Politi, Bacigaluppi et al. 2007; Suzuki, Cunningham et al. 2007; Dash, Chan et al. 2008; Himmelreich and Hoehn 2008; Kim and Song 2008; Terrovitis, Stuber et al. 2008; Wang, Wang et al. 2008; Wu, Hu et al. 2008; Kedzioreik, Ouwerkerk et al. 2009; Kim, Huh et al. 2009; Lythgoe 2009; Reekmans, Tambuyzer et al. 2009; Solanky, Chung et al. 2010; Tseng, Shih et al. 2010; Chien, Hsiao et al. 2011). Extraordinary three dimensional and anatomic resolution and high safety profile are the main advantages. To be tracked in human body and the target organ, stem cells should be enriched with a contrast agent producing a sufficient positive or negative signal to distinguish them from the non-stem cell background (Zhang and Wu 2007). The three major techniques to label stem cells for the purpose of MRI tracking are: (1) non-specific direct cell labeling, (2) indirect specific (i.e., receptor-mediated) cell labeling, which is accomplished by complexing an MR-visible contrast with a ligand that binds to a stem cell-specific receptor; and (3) reporter gene probe labeling, which is transfection with a reporter gene that expresses either an enzyme or receptor that can be detected using MRI (Gilad, Winnard et al. 2007; Kraitchman and Bulte 2008). For MRI cell labeling, direct and receptor-based labeling have been most extensively used. MRI imaging techniques are also can be divided into two groups: those generating primarily T1 contrast and T2/T2* contrast (Frangioni and Hajjar 2004). 
T1-weighted contrast agents are those that utilize the lanthanide gadolinium $(\mathrm{Gd} 3+)$, loaded via pino/endocytosis into stem cells. The main problem is that Gd3+-based contrast requires 50 - to $500-\mu \mathrm{mol} / \mathrm{L}$ concentrations of low-molecular-weight Gd3+-containing molecules or attachment to bulky scaffolds (e.g. dendrimers and dextrans) to augment the T1 effect (Frangioni and Hajjar 2004). This methods permit stem cell tracking for up to 6 weeks (Bulte, Douglas et al. 2001; Bulte, Douglas et al. 2002; Modo, Cash et al. 2002; Frangioni and Hajjar 2004).

T2/T2* contrast is by far the most commonly applied technique of stem cell imaging using MRI. Since early 1990s, different superparamagnetic iron oxide nanoparticles and more recent formulations to improve cell loading (Tat peptides) have been used (Lewin, Carlesso et al. 2000; Daldrup-Link, Rudelius et al. 2003; Frank, Miller et al. 2003; Frangioni and Hajjar 2004). Most of them are biocompatible, safe, and nontoxic, and some already have been approved by the FDA (Frangioni and Hajjar 2004). Because of the magnification effect at high field strengths, superparamagnetic nanoparticles can be used to track extremely small numbers of cells for up to several weeks.

Previous studies have confirmed that stem cell traffic throughout the body can be noninvasively monitored in MRI by labeling cells with micrometer-sized iron oxide particles or superparamagnetic iron oxides (Mancardi, Saccardi et al. 2001; Saiz, Carreras et al. 2001; Daldrup-Link, Rudelius et al. 2003; Saiz, Blanco et al. 2004; Magnitsky, Watson et al. 2005; Cheung, Chow et al. 2006; Brekke, Williams et al. 2007; Roberts, Price et al. 2007; Suzuki, Yeung et al. 2007; Arbab and Frank 2008; Kraitchman and Bulte 2008; Kraitchman, Gilson et al. 2008; Kustermann, Himmelreich et al. 2008; Mani, Adler et al. 2008; Wang, Wang et al. 2008; Chapon, Jackson et al. 2009; Sung, Hong et al. 2009; Yamada, Gurney et al. 2009; Yang, Liu et al. 2009; Drey, Ewert et al. 2010; Nohroudi, Arnhold et al. 2010; Flexman, Cross et al. 2011; Jang, Ye et al. 2011; Liu, Wang et al. 2011). Yang et al have successfully tracked stem cells following administration to animals with myocardial infarction (MI) and showed that signal attenuation is observed at MI sites for the group of cases with MI (suggesting labeled cells accumulation), while no remarkable signal attenuation is observed for the sham group (Yang, Schumacher et al. 2011). Based on such observations, it seems that the ferromagnetically labeled stem cells could provide a tool to determine whether the stem cells reach the target organ and, if so, how long they remain there. Furthermore, tracking of labeled stem cells with MRI can be used to ensure appropriate cell delivery and homing and provide insight into the preferred sites of engraftment. From this information, application of individualized stem cell therapy will be possible, as the most appropriate dosage and timing of stem cell infusion can be determined to optimize the cell-based treatment based on the clinical characteristics of each individual patient (Kraitchman and Bulte 2008).

\section{Drawbacks of MRI}

An important limitation of MRI is that it may not distinguish iron-labeled cells from free iron particles. Transfer of iron oxide particles from originally labeled cells to macrophages can lead to false interpretation of MRI data, which imposes additional disadvantage for in vivo tracking of stem cells using MRI (Jang, Ye et al. 2011). Also, some authors claim that MRI tracking of stem cells suffers from low sensitivity (Boersma, Tromp et al. 2005; Kraitchman, Tatsumi et al. 2005). In fact, initial cell-labeling techniques were hampered by limited concentration of internalized contrast agent, which lead to the limited sensitivity of MRI to detect the labeled cells (Kraitchman, Tatsumi et al. 2005). To compensate for this limited sensitivity, experimental cell-tracking studies were performed using MR imagers 
with very high magnetic field strengths of up to 14 Tesla (Weissleder, Cheng et al. 1997), which are not routinely available for clinical purposes. Schoepf et al. asserted that with clinical 1.5 Tesla MR and clinically applicable contrast agents, it is not feasible to trace the in vivo biodistribution of intravenously infused cells to more than one final target organ, or to depict the migration of the transplanted cells to several subsequent target organs over time (Schoepf, Marecos et al. 1998). Other disadvantages of MRI tracking of stem cells is the limited number of probes available (Boersma, Tromp et al. 2005) and concerns about toxic effects of MRI contrast agents on stem cells (Crabbe, Vandeputte et al. 2010; Nohroudi, Arnhold et al. 2010). It has been demonstrated that cell viability and migratory potential of stem cells are negatively correlated with incorporated magnetic particles, presumably due to interference with the actin cytoskeleton of the cells (Nohroudi, Arnhold et al. 2010). A significant limitation of MRI is that presence of some implantable devices, such as pacemakers and defibrillators, should be considered as contraindications to scanning. Although some authors suggest that patients with pacemakers can be scanned safely at $1.5 \mathrm{~T}$ (Martin, Coman et al. 2004), the role of MRI in clinical settings of stem cell tracking remains questionable, as patients with cardiac devices are undoubtedly major candidates of stem cell therapies, and cardiac MRI is not readily available at all institutions (Frangioni and Hajjar 2004).

\section{B. In vivo experiences}

Regarding the difficulties and serious complications inherent to invasive techniques of administration or delivery of stem cells to the patients' target organs, such as hemorrhage or contrast nephropathy following angiography, stem cell delivery and administration via intravenous infusion has been suggested (Detante, Moisan et al. 2009). However, there is a remarkable concern that following peripheral vein infusion of stem cells, many of cells may entrap in tissues other than the target organ, with no clinical benefit for the patient. Thus, there is a need to track stem cells after peripheral vein infusion, and to provide information about the bio-distribution of the cells delivered by such a route, in order to confirm adequate homing of the injected cells to the target organ (Gholamrezanezhad, Mirpour et al. 2011). Also these data are necessary to assess the risks of a systemic cell therapy (Detante, Moisan et al. 2009). Both animal (Gao, Dennis et al. 2001; Kraitchman, Tatsumi et al. 2005; Detante, Moisan et al. 2009) and human (Gholamrezanezhad, Mirpour et al. 2011) experiences have shown that after intravenous infusion, the labeled stem cells first accumulate in the lungs. Delivery by left ventricular cavity infusion results in drastically lower lung uptake and better uptake in the target tissue (Barbash, Chouraqui et al. 2003). During the following hours to days, the radioactivity gradually moves toward and increases in the liver and spleen. In fact, over the next 48 hours, significant proportions of cells escape from the lungs' capillaries and migrate to systemic circulation. Such an increase in spleen and liver is in contrast with the overall activity reduction which is observed in all other organs and suggests that stem cells could be sequestered in the spleen and liver (Detante, Moisan et al. 2009; Gholamrezanezhad, Mirpour et al. 2011).

\section{Future outlook and conclusion}

Although remarkable advances have been made in the development of clinically applicable stem cell tracking techniques, given the inherent limitations of currently available modalities, future investigation should focus on improving sensitivity and spatial resolution, while decreasing patient exclusion and study complexity and cost. 
At this time, no imaging modality seems to be perfect in all aspects and each modality presents its own set of advantages and disadvantages. X-ray techniques (plain radiography and $\mathrm{CT}$ ) suffer from inadequate contrast sensitivity for stem cell tracking in the clinical setting (Frangioni and Hajjar 2004). Ultrasound/echocardiography shortcomings are mainly due to their limited anatomic accessibility, resolution, and quantification, although they have the potential for single-cell detection (Frangioni and Hajjar 2004). MRI, although has the advantages of a safe profile and 3-dimensional capacity, has the lowest sensitivity $\left(10^{-3}\right.$ to $10^{-5} \mathrm{~mol} / \mathrm{L}$ ), has limited availability at some institutions and is contraindicated in patients with implantable devices (Wu 2007). Scintigraphic techniques have a fair sensitivity (10-8 to $\left.10^{-9} \mathrm{~mol} / \mathrm{L}\right)$, but are not suitable for long-term cell tracking in the case of labeling with short half life tracers (limited by the radioisotope decay) and concerns about cytotoxic effects limits the application of those radiopharmaceuticals with longer half life. Moreover, long-term tracking using scintigraphic techniques requires genetic manipulation of the stem cell, stable expression of a transgene, and multiple exposures to potentially hazardous ionizing radiation (Frangioni and Hajjar 2004). Solid-state nanotechnology modalities and reporter gene labeling, although far-flung at present, seem to be promising, as they could potentially provide noninvasive, real-time monitoring of anatomic location of single stem cells. However, concerns such as possible dispersals of QDs in the cytosol need to be resolved. Near-infrared fluorescence (10-9 to $\left.10^{-12} \mathrm{~mol} / \mathrm{L}\right)$ and bioluminescence $\left(10^{-15}\right.$ to $10^{-17}$ $\mathrm{mol} / \mathrm{L}$ ) imaging techniques are the most sensitive among the available modalities, but both are constrained to a relatively shallow tissue depth, which limits their efficacy in clinical settings. In fact, they are most suitable for small animal studies and near-surface or histological applications (Frangioni and Hajjar 2004). Reporter gene imaging, though able to evaluate cell fate by assessing the viability and proliferation of the cells more accurately, will need to concerns about its safety (because of the genetic modification) should be resolved (Zhang and $\mathrm{Wu}$ 2007).

Therefore, one should ask what biologic questions need to be addressed before selecting an appropriate imaging technique (Zhang and $\mathrm{Wu}$ 2007). As it is well summarized by Zhang, if the exact location of cell homing must be identified, then MRI is the preferred method. If only the short-term biodistribution of transplanted cells is to be determined, then scintigraphic modalities are suitable. If knowledge of the long-term fate of transplanted stem cells is sought, then reporter gene imaging is best available technique (Zhang and $\mathrm{Wu}$ 2007). As an alternative solution, multimodality imaging approaches with a tailored combination of two or more imaging techniques have been suggested, which may minimize the potential drawbacks of using each imaging modality alone and may provide the most ideal information profile for clinical applications (Zhang and Wu 2007; Higuchi, Anton et al. 2009).

\section{References}

Acton, P. D. and R. Zhou (2005). "Imaging reporter genes for cell tracking with PET and SPECT." Quarterly Journal of Nuclear Medicine and Molecular Imaging 49(4): 349360.

Aicher, A., W. Brenner, et al. (2003). "Assessment of the tissue distribution of transplanted human endothelial progenitor cells by radioactive labeling." Circulation 107(16): 2134-2139. 
Andersson, P., E. ForssellAronsson, et al. (1996). "Internalization of indium-111 into human neuroendocrine tumor cells after incubation with indium-111-DTPA-D-Phe(1)octreotide." Journal of Nuclear Medicine 37(12): 2002-2006.

Arbab, A. S. and J. A. Frank (2008). "Cellular MRI and its role in stem cell therapy." Regen Med 3(2): 199-215.

Au, W. Y., W. M. Lam, et al. (2007). "A magnetic resonance imaging study of iron overload in hemopoietic stem cell transplant recipients with increased ferritin levels." Transplantation Proceedings 39(10): 3369-3374.

Barbash, I. M., P. Chouraqui, et al. (2003). "Systemic delivery of bone marrow-derived mesenchymal stem cells to the infarcted myocardium - Feasibility, cell migration, and body distribution." Circulation 108(7): 863-868.

Barkholt, L., R. Danielsson, et al. (2003). "Indium-111 labeled donor lymphocyte infusion against liver metastases after allogeneic hematopoietic stem cell transplantation in patients with renal and colon carcinoma." Bone Marrow Transplantation 31: S148S149.

Barkholt, L., R. Danielsson, et al. (2004). "Indium-III labelled donor lymphocyte infusion via hepatic artery or IV against liver metastases of renal and colon carcinoma after allogeneic haematopoietic stem cell transplantation." Biology of Blood and Marrow Transplantation 10(2): 80-80.

Belmar, C., P. W. So, et al. (2007). "Non-invasive genetic imaging for molecular and cell therapies of cancer." Clinical \& Translational Oncology 9(11): 703-714.

Bindslev, L., M. Haack-Sorensen, et al. (2006). "Labelling of human mesenchymal stem cells with indium-111 for SPECT imaging: effect on cell proliferation and differentiation." European Journal of Nuclear Medicine and Molecular Imaging 33(10): 1171-1177.

Bindslev, L., M. Haack-Sorensen, et al. (2006). "Labelling of human mesenchymal stem cells with indium-111 for SPECT imaging: effect on cell proliferation and differentiation." Eur J Nucl Med Mol Imaging 33(10): 1171-1177.

Boersma, H. H., S. C. Tromp, et al. (2005). "Stem cell tracking: Reversing the silence of the lambs..." Journal of Nuclear Medicine 46(2): 200-203.

Bonde, J., D. J. Maxwell, et al. (2005). "In vivo imaging and tracking of defined, repopulating human stem cell subsets using fluorescence conjugated nanoparticles in a clinically applicable ex vivo protocol." Blood 106(11): 849a-849a.

Bradbury, M. S., G. Panagiotakos, et al. (2007). "Optical bioluminescence imaging of human ES cell progeny in the rodent CNS." Journal of Neurochemistry 102(6): 2029-2039.

Brekke, C., S. C. Williams, et al. (2007). "Cellular multiparametric MRI of neural stem cell therapy in a rat glioma model." Neuroimage 37(3): 769-782.

Brenner, W., A. Aicher, et al. (2004). "111In-labeled CD34+ hematopoietic progenitor cells in a rat myocardial infarction model." J Nucl Med 45(3): 512-518.

Bulte, J. W., T. Douglas, et al. (2001). "Magnetodendrimers allow endosomal magnetic labeling and in vivo tracking of stem cells." Nature Biotechnology 19(12): 1141-1147.

Bulte, J. W. M., T. Douglas, et al. (2002). "Monitoring stem cell therapy in vivo using magnetodendrimers as a new class of cellular MR contrast agents." Academic Radiology 9: S332-S335.

Cao, F., S. Lin, et al. (2006). "In vivo visualization of embryonic stem cell survival, proliferation, and migration after cardiac delivery." Circulation 113(7): 1005-1014.

Cao, Y. A., A. J. Wagers, et al. (2004). "Shifting foci of hematopoiesis during reconstitution from single stem cells." Proceedings of the National Academy of Sciences of the United States of America 101(1): 221-226. 
Chapon, C., J. S. Jackson, et al. (2009). "An in vivo multimodal imaging study using MRI and PET of stem cell transplantation after myocardial infarction in rats." Mol Imaging Biol 11(1): 31-38.

Cheung, J. S., A. M. Chow, et al. (2006). "Cell number quantification of USPIO-labeled stem cells by MRI: an in vitro study." Conf Proc IEEE Eng Med Biol Soc 1: 476-479.

Chien, L. Y., J. K. Hsiao, et al. (2011). "In vivo magnetic resonance imaging of cell tropsim, trafficking mechanism, and therapeutic impact of human mesenchymal stem cells in a murine glioma model." Biomaterials 32(12): 3275-3284.

Chin, B. B., Y. Nakamoto, et al. (2003). "111In oxine labelled mesenchymal stem cell SPECT after intravenous administration in myocardial infarction." Nucl Med Commun 24(11): 1149-1154.

Chung, S. M., T. Andersson, et al. (2002). "Analysis of different promoter systems for efficient transgene expression in mouse embryonic stem cell lines." Stem Cells 20(2): 139-145.

Correa, P. L., C. T. Mesquita, et al. (2007). "Assessment of intra-arterial injected autologous bone marrow mononuclear cell distribution by radioactive labeling in acute ischemic stroke." Clinical Nuclear Medicine 32(11): 839-841.

Crabbe, A., C. Vandeputte, et al. (2010). "Effects of MRI contrast agents on the stem cell phenotype." Cell Transplant 19(8): 919-936.

Crowder, K., J. Brant, et al. (2006). "Unique perfluorocarbon nanobeacons improve stem/progenitor cell tracking with MRI." Faseb Journal 20(4): A633-A633.

Daldrup-Link, H. E., M. Rudelius, et al. (2003). "Targeting of hematopoietic progenitor cells with MR contrast agents." Radiology 228(3): 760-767.

Dash, R., T. Chan, et al. (2008). "Magnetic Resonance Imaging with Iran-Oxide Labeling Detects Differential Cell Survival after Doxorubicin Exposure in Cardiac Myocytes, Fibroblasts, and Stem Cells." Circulation 118(18): S996-S996.

Detante, O., A. Moisan, et al. (2009). "Intravenous administration of 99mTc-HMPAO-labeled human mesenchymal stem cells after stroke: in vivo imaging and biodistribution." Cell Transplant 18(12): 1369-1379.

Doubrovin, M., I. Serganova, et al. (2004). "Multimodality in vivo molecular-genetic imaging." Bioconjugate Chemistry 15(6): 1376-1388.

Drey, F., B. Ewert, et al. (2010). "Oninvasive in vivo Tracking of Mesenchymal Stem Cells by Mri and Evaluation of Cell Therapeutic Effects." Transplant International 23: 22-22.

Flexman, J. A., D. J. Cross, et al. (2011). "Quantitative analysis of neural stem cell migration and tracer clearance in the rat brain by MRI." Mol Imaging Biol 13(1): 104-111.

Frangioni, J. V. and R. J. Hajjar (2004). "In vivo tracking of stem cells for clinical trials in cardiovascular disease." Circulation 110(21): 3378-3383.

Frangioni, J. V. and R. J. Hajjar (2004). "In vivo tracking of stem cells for clinical trials in cardiovascular disease." Circulation 110(21): 3378-3383.

Frank, J. A., B. R. Miller, et al. (2003). "Clinically applicable labeling of mammalian and stem cells by combining superparamagnetic iron oxides and transfection agents." Radiology 228(2): 480-487.

Gao, J., J. E. Dennis, et al. (2001). "The dynamic in vivo distribution of bone marrow-derived mesenchymal stem cells after infusion." Cells Tissues Organs 169(1): 12-20.

Gera, A., G. K. Steinberg, et al. (2010). "In vivo neural stem cell imaging: current modalities and future directions." Regenerative Medicine 5(1): 73-86.

Gholamrezanezhad, A., M. Bagheri, et al. (2007). "The First Experience of Stem Cell Labeling in Iran Using 111In- Oxine." Iranian Journal of Nuclear Medicine 15(2): 25-27. 
Gholamrezanezhad, A., S. Mirpour, et al. (2009). "Cytotoxicity of 111In-oxine on mesenchymal stem cells: a time-dependent adverse effect." Nucl Med Commun 30(3): 210-216.

Gholamrezanezhad, A., S. Mirpour, et al. (2011). "In vivo tracking of 111In-oxine labeled mesenchymal stem cells following infusion in patients with advanced cirrhosis." Nuclear Medicine and Biology 38(5).

Gilad, A. A., P. T. Winnard, Jr., et al. (2007). "Developing MR reporter genes: promises and pitfalls." NMR Biomed 20(3): 275-290.

Hertenstein, B., K. C. Wollert, et al. (2004). "Monitoring of bone marrow cell homing in the infarcted human myocardium by PET." Blood 104(11): 736a-736a.

Higuchi, T., M. Anton, et al. (2009). "Combined Reporter Gene PET and Iron Oxide MRI for Monitoring Survival and Localization of Transplanted Cells in the Rat Heart." Journal of Nuclear Medicine 50(7): 1088-1094.

Hill, J. M., A. J. Dick, et al. (2003). "Serial cardiac magnetic resonance imaging of injected mesenchymal stem cells." Circulation 108(8): 1009-1014.

Himmelreich, U. and M. Hoehn (2008). "Stem cell labeling for magnetic resonance imaging." Minimally Invasive Therapy \& Allied Technologies 17(2): 132-142.

Hoehn, M., E. Kustermann, et al. (2002). "Monitoring of implanted stem cell migration in vivo: A highly resolved in vivo magnetic resonance imaging investigation of experimental stroke in rat." Proceedings of the National Academy of Sciences of the United States of America 99(25): 16267-16272.

Hofmann, M., K. C. Wollert, et al. (2005). "Monitoring of bone marrow cell homing into the infarcted human myocardium." Circulation 111(17): 2198-2202.

Hsiao, J. K., M. F. Tai, et al. (2007). "Magnetic nanoparticle labeling of mesenchymal stem cells without transfection agent: Cellular behavior and capability of detection with clinical 1.5 T magnetic resonance at the single cell level." Magnetic Resonance in Medicine 58(4): 717-724.

Hsieh, S. C., F. F. Wang, et al. (2006). "The inhibition of osteogenesis with human bone marrow mesenchymal stem cells by CdSe/ZnS quantum dot labels." Biomaterials 27(8): 1656-1664.

Jaiswal, J. K., H. Mattoussi, et al. (2003). "Long-term multiple color imaging of live cells using quantum dot bioconjugates." Nature Biotechnology 21(1): 47-51.

Jakobsson, J., N. Rosenqvist, et al. (2004). "Dynamics of transgene expression in a neural stem cell line transduced with lentiviral vectors incorporating the cHS4 insulator." Experimental Cell Research 298(2): 611-623.

Jang, Y. Y., Z. Ye, et al. (2011). "Molecular imaging and stem cell research." Mol Imaging 10(2): 111-122.

Jansen, J. F. A., M. J. Shamblott, et al. (2006). "Stem cell profiling by nuclear magnetic resonance spectroscopy." Magnetic Resonance in Medicine 56(3): 666-670.

Josephson, L., M. F. Kircher, et al. (2002). "Near-infrared fluorescent nanoparticles as combined MR/optical imaging probes." Bioconjugate Chemistry 13(3): 554-560.

Kang, W. J., H. J. Kang, et al. (2006). "Tissue distribution of 18F-FDG-labeled peripheral hematopoietic stem cells after intracoronary administration in patients with myocardial infarction." J Nucl Med 47(8): 1295-1301.

Kedzioreik, D., R. Ouwerkerk, et al. (2009). "X-Ray- and MRI-Visible Microencapsulated Mesenchymal Stem Cell for Cell Delivery and Tracking on Clinical Scanners." Journal of Nuclear Medicine 50(4): 657-657.

Kim, D. and J. Song (2008). "Stem cell tracking using magnetic resonance imaging." Tissue Engineering and Regenerative Medicine 5(3): 420-424. 
Kim, Y. J., Y. M. Huh, et al. (2009). "In vivo magnetic resonance imaging of injected mesenchymal stem cells in rat myocardial infarction; simultaneous cell tracking and left ventricular function measurement." International Journal of Cardiovascular Imaging 25: 99-109.

Klibanov, A. L., P. T. Rasche, et al. (2004). "Detection of individual microbubbles of ultrasound contrast agents: imaging of free-floating and targeted bubbles." Invest Radiol 39(3): 187-195.

Klug, M. G., M. H. Soonpaa, et al. (1996). "Genetically selected cardiomyocytes from differentiating embryonic stem cells form stable intracardiac grafts." Journal of Clinical Investigation 98(1): 216-224.

Kraitchman, D. L. and J. W. Bulte (2009). "In vivo imaging of stem cells and Beta cells using direct cell labeling and reporter gene methods." Arterioscler Thromb Vasc Biol 29(7): 1025-1030.

Kraitchman, D. L. and J. W. M. Bulte (2008). "Imaging of stem cells using MRI." Basic Research in Cardiology 103(2): 105-113.

Kraitchman, D. L. and J. W. M. Bulte (2009). "In vivo Imaging of Stem Cells and Beta Cells Using Direct Cell Labeling and Reporter Gene Methods." Arteriosclerosis Thrombosis and Vascular Biology 29(7): 1025-U1103.

Kraitchman, D. L., W. D. Gilson, et al. (2008). "Stem cell therapy: MRI guidance and monitoring." J Magn Reson Imaging 27(2): 299-310.

Kraitchman, D. L., M. Tatsumi, et al. (2005). "Dynamic imaging of allogeneic mesenchymal stem cells trafficking to myocardial infarction." Circulation 112(10): 1451-1461.

Kraitchman, D. L., M. Tatsumi, et al. (2005). "Dynamic imaging of allogeneic mesenchymal stem cells trafficking to myocardial infarction." Circulation 112(10): 1451-1461.

Kustermann, E., U. Himmelreich, et al. (2008). "Efficient stem cell labeling for MRI studies." Contrast Media Mol Imaging 3(1): 27-37.

Kustermann, E., W. Roell, et al. (2005). "Stem cell implantation in ischemic mouse heart: a high-resolution magnetic resonance imaging investigation." Nmr in Biomedicine 18(6): 362-370.

Lavender, J. P., J. M. Goldman, et al. (1977). "Kinetics of indium-III labelled lymphocytes in normal subjects and patients with Hodgkin's disease." Br Med J 2(6090): 797-799.

Legrand, J. F., O. Cougnenc, et al. (2007). "Evaluation of a method of stem cell labelling with Indium-111 oxine: effects on viability and proliferation." European Journal of Nuclear Medicine and Molecular Imaging 34: S284-S284.

Lewin, M., N. Carlesso, et al. (2000). "Tat peptide-derivatized magnetic nanoparticles allow in vivo tracking and recovery of progenitor cells." Nature Biotechnology 18(4): 410414.

Li, Z., B. Fehse, et al. (2002). "Persisting multilineage transgene expression in the clonal progeny of a hematopoietic stem cell." Leukemia 16(9): 1655-1663.

Li, Z. J., Y. Suzuki, et al. (2008). "Comparison of reporter gene and iron particle labeling for tracking fate of human embryonic stem cells and differentiated endothelial cells in living subjects." Stem Cells 26(4): 864-873.

Lin, S., X. Xie, et al. (2007). "Quantum dot imaging for embryonic stem cells." BMC Biotechnol 7: 67.

Liu, G., Z. Wang, et al. (2011). "Low molecular weight alkyl-polycation wrapped magnetite nanoparticle clusters as MRI probes for stem cell labeling and in vivo imaging." Biomaterials 32(2): 528-537.

Lu, S., X. Xu, et al. (2010). "Targeting of Embryonic Stem Cells by Peptide-Conjugated Quantum Dots." Plos One 5(8): -. 
Lythgoe, M. F. (2009). "High-field magnetic resonance imaging: stem cell tracking and phenotyping of transgenic mice." Neurogastroenterology and Motility 21(2): V-V.

Magnitsky, S., R. M. Walton, et al. (2007). "Magnetic resonance imaging as a tool for monitoring stem cell migration." Neurodegenerative Diseases 4(4): 314-321.

Magnitsky, S., D. J. Watson, et al. (2005). "In vivo and ex vivo MRI detection of localized and disseminated neural stem cell grafts in the mouse brain." Neuroimage 26(3): 744754 .

Mancardi, G. L., R. Saccardi, et al. (2001). "Autologous hematopoietic stem cell transplantation suppresses Gd-enhanced MRI activity in MS." Neurology 57(1): 6268.

Mani, V., E. Adler, et al. (2008). "Serial in vivo positive contrast MRI of iron oxide-labeled embryonic stem cell-derived cardiac precursor cells in a mouse model of myocardial infarction." Magn Reson Med 60(1): 73-81.

Martin, E. T., J. A. Coman, et al. (2004). "Magnetic resonance imaging and cardiac pacemaker safety at 1.5-Tesla." Journal of the American College of Cardiology 43(7): 1315-1324.

Maskali, F., N. Tran, et al. (2004). "Indium-111 oxine labeling of rat bone marrow-derived mesenchymal stem cells for in vivo imaging during autologous cell therapy of myocardial infarction." European Journal of Nuclear Medicine and Molecular Imaging 31: S204-S204.

Maskali, F., N. Tran, et al. (2005). "Indium-111 oxine labeling of rat bone marrow-derived mesenchymal stem cells for in vivo imaging during autologous cell therapy of myocardial infarction." Journal of the American College of Cardiology 45(3): 227a227a.

McColgan, P., P. Sharma, et al. (2011). "Stem Cell Tracking in Human Trials: A MetaRegression." Stem Cell Rev.

Meyerwittkopf, M., A. Bockisch, et al. (1993). "Examination in-Vivo of Cell-Migration of Tc99m Hmpao Labeled Transplanted Liver Stem-Cells in the Rabbit." Archives of Gynecology and Obstetrics 254(1-4): 1059-1061.

Min, J. J., Y. Ahn, et al. (2006). "In vivo bioluminescence imaging of cord blood derived mesenchymal stem cell transplantation into rat myocardium." Annals of Nuclear Medicine 20(3): 165-170.

Min, J. J., U. C. N. Le, et al. (2008). "Visualization of Embryonic Stem Cell Survival In vivo by Adenoviral-driven Bioluminescence Reporter in Rat Corpus Cavernosum." Tissue Engineering and Regenerative Medicine 5(4-6): 743-749.

Modo, M., D. Cash, et al. (2002). "Tracking transplanted stem cell migration using bifunctional, contrast agent-enhanced, magnetic resonance imaging." Neuroimage 17(2): 803-811.

Nakayama, A., F. del Monte, et al. (2002). "Functional near-infrared fluorescence imaging for cardiac surgery and targeted gene therapy." Mol Imaging 1(4): 365-377.

Neirinckx, R. D., J. F. Burke, et al. (1988). "The retention mechanism of technetium-99m-HMPAO: intracellular reaction with glutathione." J Cereb Blood Flow Metab 8(6): S4-12.

Nohroudi, K., S. Arnhold, et al. (2010). "In vivo MRI stem cell tracking requires balancing of detection limit and cell viability." Cell Transplant 19(4): 431-441.

Nohroudi, K., S. Arnhold, et al. (2010). "In vivo MRI Stem Cell Tracking Requires Balancing of Detection Limit and Cell Viability." Cell Transplantation 19(4): 431-441.

Nyolczas, N., S. Charwat, et al. (2009). "Tracking the migration of cardially delivered therapeutic stem cells in vivo: state of the art." Regenerative Medicine 4(3): 407-422. 
Olmer, R., A. Haase, et al. (2009). "Human Oct3/4 Promoter Dependent Lentiviral Transgene Expression for Simplified Monitoring of Primate Embryonic Stem Cell Culture." Tissue Engineering Part A 15(3): 727-727.

Park, K. S., J. Tae, et al. (2010). "Characterization, in vitro cytotoxicity assessment, and in vivo visualization of multimodal, RITC-labeled, silica-coated magnetic nanoparticles for labeling human cord blood-derived mesenchymal stem cells." Nanomedicine 6(2): 263-276.

Partlow, K. C., J. J. Chen, et al. (2007). "F-19 magnetic resonance imaging for stem/ progenitor cell tracking with multiple unique perfluorocarbon nanobeacons." Faseb Journal 21(8): 1647-1654.

$\mathrm{Pi}, \mathrm{Q}$. M., W. J. Zhang, et al. (2010). "Degradation or excretion of quantum dots in mouse embryonic stem cells." Bmc Biotechnology 10: -.

Politi, L. S., M. Bacigaluppi, et al. (2007). "Magnetic resonance-based tracking and quantification of intravenously injected neural stem cell accumulation in the brains of mice with experimental multiple sclerosis." Stem Cells 25(10): 2583-2592.

Polzer, H., F. Haasters, et al. (2010). "Quantification of Fluorescence Intensity of Labeled Human Mesenchymal Stem Cells and Cell Counting of Unlabeled Cells in PhaseContrast Imaging: An Open-Source-Based Algorithm." Tissue Engineering Part CMethods 16(6): 1277-1285.

Pomper, M. G., H. Hammond, et al. (2009). "Serial imaging of human embryonic stem-cell engraftment and teratoma formation in live mouse models." Cell Research 19(3): 370-379.

Ramot, Y., M. Steiner, et al. (2010). "Pulmonary thrombosis in the mouse following intravenous administration of quantum dot-labeled mesenchymal cells." Nanotoxicology 4(1): 98-105.

Reekmans, K., B. Tambuyzer, et al. (2009). "Combined in vivo Bioluminescence and Magnetic Resonance Imaging of Adherently Cultured Neural Stem/Progenitor Cell Implants in Brain of Mice." Glia 57(13): S76-S76.

Reumers, V., C. M. Deroose, et al. (2008). "Noninvasive and quantitative monitoring of adult neuronal stem cell migration in mouse brain using bioluminescence imaging." Stem Cells 26(9): 2382-2390.

Reynolds, J. S., T. L. Troy, et al. (1999). "Imaging of spontaneous canine mammary tumors using fluorescent contrast agents." Photochemistry and Photobiology 70(1): 87-94.

Roberts, T. J., J. Price, et al. (2007). "Pharmacological MRI of stem cell transplants in the 3nitropropionic acid-damaged striatum." Neuroscience 144(1): 100-109.

Rodriguez-Porcel, M., O. Gheysens, et al. (2005). "Image-guided cardiac cell delivery using high-resolution small-animal ultrasound." Molecular Therapy 12(6): 1142-1147.

Roelants, V., D. Labar, et al. (2008). "Comparison Between Adenoviral and Retroviral Vectors for the Transduction of the Thymidine Kinase PET Reporter Gene in Rat Mesenchymal Stem Cells." Journal of Nuclear Medicine 49(11): 1836-1844.

Ruan, J., J. Shen, et al. (2010). "Viability and pluripotency studying of human embryo stem cells labeled with quantum dots." Nano Biomedicine and Engineering 2(4): 245-251.

Rutten, M., M. Janes, et al. (2010). "Comparison of Quantum Dots and CM-DiI for Labeling Porcine Autologous Bone Marrow Mononuclear Progenitor Cells." The Open Stem Cell Journal 2: 25-36.

Saiz, A., Y. Blanco, et al. (2004). "Clinical and MRI outcome after autologous hematopoietic stem cell transplantation in MS." Neurology 62(2): 282-284.

Saiz, A., E. Carreras, et al. (2001). "MRI and CSF oligoclonal bands after autologous hematopoietic stem cell transplantation in MS." Neurology 56(8): 1084-1089. 
Schoepf, U., E. M. Marecos, et al. (1998). "Intracellular magnetic labeling of lymphocytes for in vivo trafficking studies." Biotechniques 24(4): 642-+.

Serganova, I. and R. Blasberg (2005). "Reporter gene imaging: potential impact on therapy." Nuclear Medicine and Biology 32(7): 763-780.

Shah, K. (2005). "Current advances in molecular imaging of gene and cell therapy for cancer." Cancer Biology \& Therapy 4(5): 518-523.

Sheikh, A. Y., S. A. Lin, et al. (2007). "Molecular imaging of bone marrow mononuclear cell homing and engraftment in ischemic myocardium." Stem Cells 25(10): 2677-2684.

Solanky, B., Y. L. Chung, et al. (2010). "Characterization of a Human Neural Stem Cell Biomarker Using Magnetic Resonance Spectroscopy." Cell Transplantation 19(3): 362-363.

Steindler, D. A. (2007). "Stem cells, regenerative medicine, and animal models of disease." Ilar Journal 48(4): 323-338.

Sung, C. K., K. A. Hong, et al. (2009). "Dual-Modal Nanoprobes for Imaging of Mesenchymal Stem Cell Transplant by MRI and Fluorescence Imaging." Korean Journal of Radiology 10(6): 613-622.

Sung, C. K., K. A. Hong, et al. (2009). "Dual-modal nanoprobes for imaging of mesenchymal stem cell transplant by MRI and fluorescence imaging." Korean J Radiol 10(6): 613622.

Suzuki, Y., C. H. Cunningham, et al. (2007). "In vivo magnetic resonance imaging of stem cell viability." Journal of the American College of Cardiology 49(9): 158a-158a.

Suzuki, Y., A. C. Yeung, et al. (2007). "Cardiovascular MRI for stem cell therapy." Curr Cardiol Rep 9(1): 45-50.

Tamura, M., K. Unno, et al. (2004). "In vivo trafficking of endothelial progenitor cells and their possible involvement in tumor neovascularization." Life Sciences 75(5): 575584 .

Tang, Y., K. Shah, et al. (2003). "In vivo tracking of neural progenitor cell migration to glioblastomas." Hum Gene Ther 14(13): 1247-1254.

Tepper-Wessels, K., M. Loeschinger, et al. (2001). "Detection of rare stem cell populations from umbilical cord blood by multi fluorescence imaging." Molecular Biology of the Cell 12: 134a-134a.

Terrovitis, J., M. Stuber, et al. (2008). "Magnetic resonance imaging overestimates ferumoxide-labeled stem cell survival after transplantation in the heart." Circulation 117(12): 1555-1562.

Tran, N. D., D. Lair, et al. (2010). "Transgene Independent Germ Cell Differentiation From Human Embryonic Stem Cells (hESCs)." Fertility and Sterility 93(5): S22-S22.

Tseng, C. L., I. L. Shih, et al. (2010). "Gadolinium hexanedione nanoparticles for stem cell labeling and tracking via magnetic resonance imaging." Biomaterials 31(20): 54275435.

van den Bos, E. J., A. Wagner, et al. (2003). "Improved efficacy of stem cell labeling for magnetic resonance imaging studies by the use of cationic liposomes." Cell Transplantation 12(7): 743-756.

Wang, X. L., M. Rosol, et al. (2003). "Dynamic tracking of human hematopoietic stem cell engraftment using in vivo bioluminescence imaging." Blood 102(10): 3478-3482.

Wang, Y. X., H. H. Wang, et al. (2008). "Pitfalls in employing superparamagnetic iron oxide particles for stem cell labelling and in vivo MRI tracking." Br J Radiol 81(972): 987988. 
Wang, Y. X. J., H. H. Wang, et al. (2008). "Pitfalls in employing superparamagnetic iron oxide particles for stem cell labelling and in vivo MRI tracking." British Journal of Radiology 81(972): 987-988.

Weissleder, R., H. C. Cheng, et al. (1997). "Magnetically labeled cells can be detected by MR imaging." Jmri-Journal of Magnetic Resonance Imaging 7(1): 258-263.

Weldon, M. J., A. E. Joseph, et al. (1995). "Comparison of 99m technetium hexamethylpropylene-amine oxime labelled leucocyte with 111-indium tropolonate labelled granulocyte scanning and ultrasound in the diagnosis of intra-abdominal abscess." Gut 37(4): 557-564.

Wickline, S. A. and G. M. Lanza (2003). "Nanotechnology for molecular imaging and targeted therapy." Circulation 107(8): 1092-1095.

Wollert, K. C., M. Hofmann, et al. (2004). "Monitoring of bone marrow cell homing to the infarcted human myocardium." Circulation 110(17): 436-436.

$\mathrm{Wu}, \mathrm{J}$. C. (2007). "Comparison of imaging techniques for tracking cardiac stem cell therapy." Journal of Nuclear Medicine 48(12): 1916-1919.

Wu, J. C., I. Y. Chen, et al. (2003). "Molecular imaging of cardiac cell transplantation in living animals using optical bioluminescence and positron emission tomography." Circulation 108(11): 1302-1305.

$\mathrm{Wu}, \mathrm{X} ., \mathrm{J}$. Hu, et al. (2008). "In vivo tracking of superparamagnetic iron oxide nanoparticlelabeled mesenchymal stem cell tropism to malignant gliomas using magnetic resonance imaging." Journal of Neurosurgery 108(2): 320-329.

Yamada, M., P. T. Gurney, et al. (2009). "Manganese-guided cellular MRI of human embryonic stem cell and human bone marrow stromal cell viability." Magn Reson Med 62(4): 1047-1054.

Yang, J., J. Liu, et al. (2009). "In vivo MRI of endogenous stem/progenitor cell migration from subventricular zone in normal and injured developing brains." Neuroimage 48(2): 319-328.

Yang, Y., A. Schumacher, et al. (2011). "Monitoring bone marrow-originated mesenchymal stem cell traffic to myocardial infarction sites using magnetic resonance imaging." Magn Reson Med.

Yukawa, H., Y. Kagami, et al. (2010). "Quantum dots labeling using octa-arginine peptides for imaging of adipose tissue-derived stem cells." Biomaterials 31(14): 4094-4103.

Zhang, H. L., H. Qiao, et al. (2011). "Utility of Dual-modality Bioluminescence and MRI in Monitoring Stem Cell Survival and Impact on Post Myocardial Infarct Remodeling." Academic Radiology 18(1): 3-12.

Zhang, S. J. and J. C. Wu (2007). "Comparison of imaging techniques for tracking cardiac stem cell therapy." J Nucl Med 48(12): 1916-1919. 


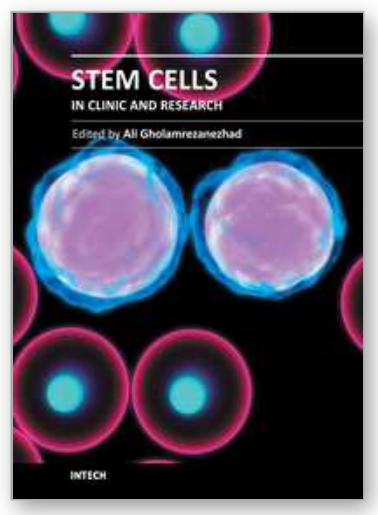

\author{
Stem Cells in Clinic and Research \\ Edited by Dr. Ali Gholamrezanezhad
}

ISBN 978-953-307-797-0

Hard cover, 804 pages

Publisher InTech

Published online 23, August, 2011

Published in print edition August, 2011

Based on our current understanding of cell biology and strong supporting evidence from previous experiences, different types of human stem cell populations are capable of undergoing differentiation or trans-differentiation into functionally and biologically active cells for use in therapeutic purposes. So far, progress regarding the use of both in vitro and in vivo regenerative medicine models already offers hope for the application of different types of stem cells as a powerful new therapeutic option to treat different diseases that were previously considered to be untreatable. Remarkable achievements in cell biology resulting in the isolation and characterization of various stem cells and progenitor cells has increased the expectation for the development of a new approach to the treatment of genetic and developmental human diseases. Due to the fact that currently stem cells and umbilical cord banks are so strictly defined and available, it seems that this mission is investigationally more practical than in the past. On the other hand, studies performed on stem cells, targeting their conversion into functionally mature tissue, are not necessarily seeking to result in the clinical application of the differentiated cells; In fact, still one of the important goals of these studies is to get acquainted with the natural process of development of mature cells from their immature progenitors during the embryonic period onwards, which can produce valuable results as knowledge of the developmental processes during embryogenesis. For example, the cellular and molecular mechanisms leading to mature and adult cells developmental abnormalities are relatively unknown. This lack of understanding stems from the lack of a good model system to study cell development and differentiation. Hence, the knowledge reached through these studies can prove to be a breakthrough in preventing developmental disorders. Meanwhile, many researchers conduct these studies to understand the molecular and cellular basis of cancer development. The fact that cancer is one of the leading causes of death throughout the world, highlights the importance of these researches in the fields of biology and medicine.

\title{
How to reference
}

In order to correctly reference this scholarly work, feel free to copy and paste the following:

Sahar Mirpour and Ali Gholamrezanezhad (2011). Clinical Stem Cell Imaging and In vivo Tracking, Stem Cells in Clinic and Research, Dr. Ali Gholamrezanezhad (Ed.), ISBN: 978-953-307-797-0, InTech, Available from: http://www.intechopen.com/books/stem-cells-in-clinic-and-research/clinical-stem-cell-imaging-and-in-vivotracking

\section{INTECH}

open science | open minds 


\section{InTech Europe}

University Campus STeP Ri

Slavka Krautzeka 83/A

51000 Rijeka, Croatia

Phone: +385 (51) 770447

Fax: +385 (51) 686166

www.intechopen.com

\section{InTech China}

Unit 405, Office Block, Hotel Equatorial Shanghai

No.65, Yan An Road (West), Shanghai, 200040, China

中国上海市延安西路65号上海国际贵都大饭店办公楼 405 单元

Phone: +86-21-62489820

Fax: $+86-21-62489821$ 
(C) 2011 The Author(s). Licensee IntechOpen. This chapter is distributed under the terms of the Creative Commons Attribution-NonCommercialShareAlike-3.0 License, which permits use, distribution and reproduction for non-commercial purposes, provided the original is properly cited and derivative works building on this content are distributed under the same license. 